International Journal of Bifurcation and Chaos, Vol. 7, No. 8 (1997) 1873-1885

(C) World Scientific Publishing Company

\title{
$n$-DOUBLE SCROLL HYPERCUBES IN 1-D CNNs
}

\author{
J. A. K. SUYKENS* \\ Katholieke Universiteit Leuven, Department of Electrical Engineering, ESAT-SISTA, \\ Kardinaal Mercierlaan 94, B-3001 Leuven (Heverlee), Belgium \\ L. O. $\mathrm{CHUA}^{\dagger}$ \\ Department of Electrical Engineering and Computer Science \\ University of California at Berkeley, Berkeley, CA 94720, USA
}

Received October 7, 1996; Revised October 26, 1996

\begin{abstract}
Unidirectional and diffusive coupling of identical $n$-double scroll cells in a one-dimensional cellular neural network is studied. Weak coupling between the cells leads to hyperchaos, with $n$-double scroll hypercube attractors observed in the common state subspace of the cells. Individually the cells remain behaving as $n$-double scrolls. The $n$-double scroll hypercubes are filled with multiple scrolls. Their birth goes through the mechanism of intermittency.
\end{abstract}

\section{Introduction}

Among the many generalizations proposed to Chua's circuit (see e.g. [Chua, 1994; Madan, 1993]), the one introduced by Suykens and Vandewalle [1993] considers additional breakpoints in the nonlinear characteristic in order to generate multiple scrolls, resulting into so-called $n$-double scroll attractors, where $n$ is a natural number. The well-known double scroll corresponds to a 1-double scroll within this framework. An electrical circuit implementation has been made by Arena et al. [1996] which confirms the existence of $n$-double scrolls. Moreover this implementation makes use of a piecewise linear representation of the nonlinear characteristic.

On the other hand many interesting phenomena have been observed by taking Chua's circuits as cells in Cellular Neural Networks (CNNs) (see e.g. [Chua \& Roska, 1993; Chua et al., 1995]), with, for example, unidirectional or diffusive coupling between the cells. One-dimensional CNNs with diffusive coupling have been investigated in [Zheleznyak \& Chua, 1994; Pivka, 1995; Nekorkin \& Chua, 1993] with phenomena occurring such as spatiotemporal chaos, spatial disorder and traveling waves. In two-dimensional CNNs phenomena such as traveling waves, spiral waves, trigger and target waves and Turing patterns have been observed [Pérez-Muñuzuri et al., 1993; Pivka, 1995; Chua \& Goraş, 1995]. Scroll waves and scroll rings can be generated in three-dimensional CNNs with diffusive coupling [Pivka et al., 1995]. Unidirectional coupling in one-dimensional CNNs has been studied in [Kapitaniak et al., 1994; Kapitaniak \& Chua, 1994].

In the latter work of Kapitaniak \& Chua, hyperchaotic behavior has been generated by coupling identical Chua's circuits that behave individually as double scrolls. In the common state subspace of the cells the birth of a so-called double-double scroll attractor has been observed. The work reported in our paper is very much related to this result. Taking a 1D-CNN consisting of $n$-double scroll cells

\footnotetext{
*E-mail: johan.suykens@esat.kuleuven.ac.be
}

${ }^{\dagger}$ E-mail: chua@fred.eecs.berkeley.edu 
with weak unidirectional or diffusive coupling between the cells, an $n$-double scroll hypercube is obtained in the common state subspace of the cells. The dimension of this hypercube is equal to the number of cells in the array. The number of scrolls within the hypercube increases with the number $n$ of the $n$-double scroll cell. For the arrays that have been simulated, the number of positive Lyapunov exponents is equal to the number of cells, which has been stated also in [Kapitaniak \& Chua, 1994]. The birth of the double-double scroll attractor in [Kapitaniak \& Chua, 1994] goes by means of classical intermittency, with occasional bursts from a three-dimensional manifold to higher dimensions. A similar mechanism is at the basis of the birth of the $n$-double scroll hypercube.

An engineering motivation for studying higher dimensional hyperchaotic arrays is in, for instance, their use for secure communication applications (see e.g. [Wu \& Chua, 1994]). CNNs consisting of $n$ double scroll circuits can be represented as Lur'e systems for which a condition of master-slave synchronization is given in [Suykens \& Vandewalle, 1996]. The latter condition is based on a Lur'ePostnikov Lyapunov function for the error system and is expressed as a matrix inequality.

This paper is organized as follows. In Sec. 2 we review the equations of $n$-double scroll circuits. In Sec. 3 1-D CNNs with unidirectional and diffusive coupling of $n$-double scroll cells are described. In Sec. 4 the existence of $n$-double scroll hypercubes for weak coupling is reported. In Sec. 5 the route from synchronization to hypercubes is discussed.

\section{2. $n$-Double Scroll Circuits}

The electrical circuit for obtaining $n$-double scrolls, according to the implementation of Arena et al. [1996], is given by

$$
\left\{\begin{array}{l}
\dot{x}=\alpha[y-h(x)] \\
\dot{y}=x-y+z \\
\dot{z}=-\beta y
\end{array}\right.
$$

with as nonlinear function the piecewise linear characteristic

$$
\begin{aligned}
h(x)= & m_{2 n-1} x \\
& +\frac{1}{2} \sum_{i=1}^{2 n-1}\left(m_{i-1}-m_{i}\right)\left(\left|x+c_{i}\right|-\left|x-c_{i}\right|\right),
\end{aligned}
$$

consisting of $2(2 n-1)$ breakpoints, where $n$ is a natural number. In order to generate $n$-double scrolls one takes $\alpha=9$ and $\beta=14.286$. Some special cases are:

- 1-double scroll:

$$
\begin{gathered}
m_{0}=-1 / 7 \quad m_{1}=2 / 7 \\
c_{1}=1
\end{gathered}
$$

corresponding to the well-known double scroll (see e.g. [Chua et al. 1986; Chua, 1994; Madan, 1993]).

- 2-double scroll:

$$
\begin{gathered}
m_{0}=-1 / 7 \quad m_{1}=2 / 7 \quad m_{2}=-4 / 7 \quad m_{3}=m_{1} \\
c_{1}=1 \quad c_{2}=2.15 \quad c_{3}=3.6
\end{gathered}
$$

- 3-double scroll:

$$
\begin{array}{rlll}
m_{0}=-1 / 7 & m_{1}=2 / 7 & m_{2}=-4 / 7 \\
m_{3}=m_{1} & m_{4}=m_{2} & m_{5}=m_{3} \\
c_{1}=1 & c_{2}=2.15 & c_{3}=3.6 & c_{4}=8.2 \quad c_{5}=13 .
\end{array}
$$

The 2-double scroll and 3-double scroll are shown in Fig. 1. For more details about $n$-double scrolls we refer to [Suykens \& Vandewalle, 1993; Arena et al. 1996].

\section{One-Dimensional CNNs with $n$-Double Scroll Cells}

Let us now consider the following one-dimensional cellular neural network (CNN) consisting of identical $n$-double scroll cells, with either unidirectional coupling between the cells

$$
\left\{\begin{array}{l}
\dot{x}^{(j)}=\alpha\left[y^{(j)}-h\left(x^{(j)}\right)\right] \\
\dot{y}^{(j)}=x^{(j)}-y^{(j)}+z^{(j)}+K_{j-1}\left(y^{(j)}-y^{(j-1)}\right) \\
\dot{z}^{(j)}=-\beta y^{(j)}, \quad j=1,2, \ldots, L
\end{array}\right.
$$

or diffusive coupling as

$$
\left\{\begin{aligned}
\dot{x}^{(j)}= & \alpha\left[y^{(j)}-h\left(x^{(j)}\right)\right] \\
& +D_{x}\left(x^{(j-1)}-2 x^{(j)}+x^{(j+1)}\right) \\
\dot{y}^{(j)}= & x^{(j)}-y^{(j)}+z^{(j)} \\
\dot{z}^{(j)}= & -\beta y^{(j)}, \quad j=1,2, \ldots, L
\end{aligned}\right.
$$




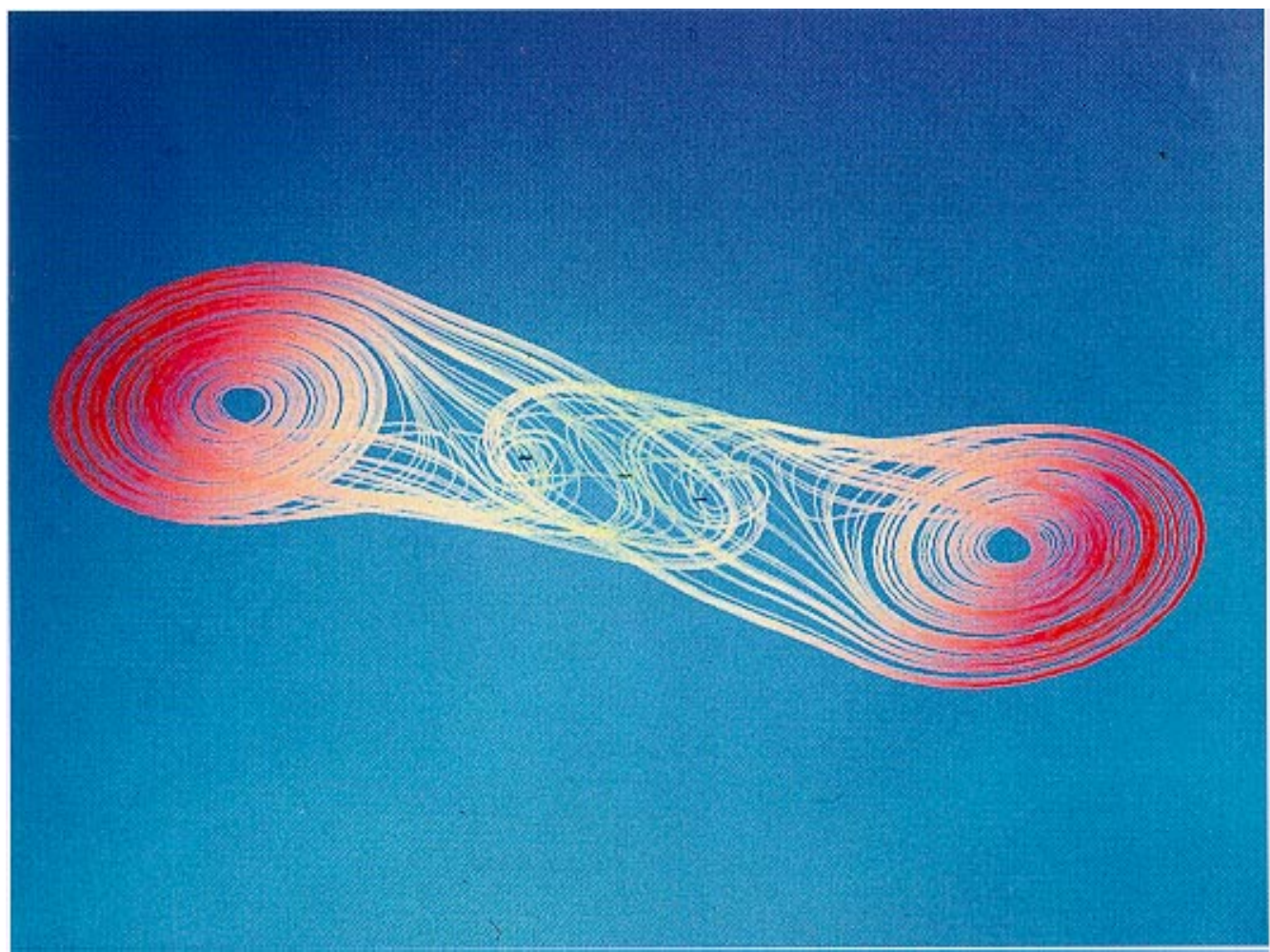

(a)

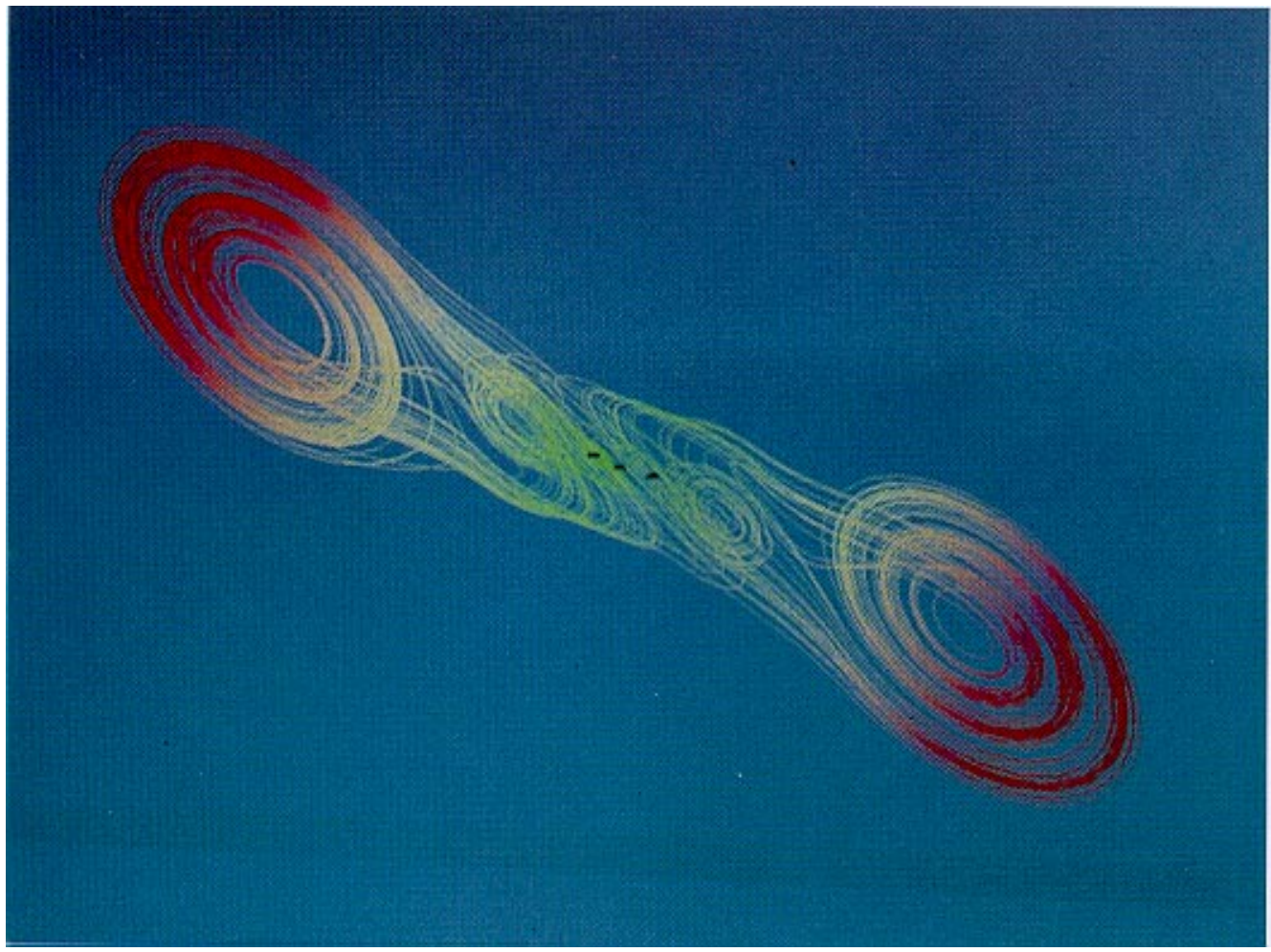

(b)

Fig. 1. (a) Three-dimensional view on the 2-double scroll attractor; (b) three-dimensional view on the 3-double scroll attractor. 
or as

$$
\left\{\begin{aligned}
\dot{x}^{(j)}= & \alpha\left[y^{(j)}-h\left(x^{(j)}\right)\right] \\
\dot{y}^{(j)}= & x^{(j)}-y^{(j)}+z^{(j)} \\
& +D_{y}\left(x^{(j-1)}-2 x^{(j)}+x^{(j+1)}\right) \\
\dot{z}^{(j)}= & -\beta y^{(j)}, \quad j=1,2, \ldots, L
\end{aligned}\right.
$$

where $L$ denotes the number of cells. We impose the conditions $y^{(0)}=y^{(L)}$ for Eq. (3) and $x^{(0)}=x^{(L)}$, $x^{(L+1)}=x^{(1)}$ for Eqs. (4) and (5). For the coupling constants, $K_{0}=0, K_{j}=K(j=1, \ldots, L-1)$ and positive diffusion coefficients $D_{x}, D_{y}$ are chosen.

The scheme Eq. (3) with unidirectional coupling has been studied by Kapitaniak \& Chua [1994] for the case $n=1$ with Chua's circuits as cells. The other schemes of 1-D reaction-diffusion CNNs has been described in [Chua et al. 1995; Pivka, 1995; Zheleznyak \& Chua, 1994].

\section{Weak Coupling: $n$-Double Scroll Hypercubes}

Let us consider first the case $n=2$ with 2-double scroll circuits in the CNNs [Eqs. (3) and (5)]. Both for weak unidirectional and diffusive coupling one obtains a 2-double scroll hypercube in the common state subspace of the cells, i.e. in $\left(x^{(1)}, x^{(2)}\right)$ for $L=$ $2 ;\left(x^{(1)}, x^{(2)}, x^{(3)}\right)$ for $L=3 ;\left(x^{(1)}, x^{(2)}, x^{(3)}, x^{(4)}\right)$ for $L=4$, etc. The dimension of the hypercube is equal to the number of cells $L$. So one obtains for $L=2,3,4$ a 2-double scroll square, cube and hypercube, respectively (see Figs. 2 and 3). This phenomenon is typically obtained for 'small' values of $K, D_{x}$ or $D_{y}$, such as 0.01 or 0.001 . For this weak coupling, the individual cells remain behaving as 2double scrolls while the hypercube is formed in the common cell-space (Fig. 4). Simulation results for 10 cells with the formation of a ten-dimensional hypercube are shown in Fig. 5. The initial states were chosen 'close' to the origin (according to [Kapitaniak \& Chua, 1994]): $x^{(j)}=0.01+(j-1) 0.001$, $y^{(j)}=0, z^{(j)}=0$ for $j=1,2, \ldots, L$ and $L=2,3$, 4, 10. Similar phenomena were obtained for other choices of the initial state.

For the case $n=3$ with 3 -double scroll cells in the CNNs [Eqs. (3) and (4)], similar phenomena occur. 3-Double scroll squares, cubes and hypercubes, obtained for $L=2,3,4$, respectively are shown in Figs. 6 and 7. The larger the number of $n$ the more scrolls the $L$-dimensional hypercube con- tains. The same initial states were chosen as for the corresponding $n=2$ cases.

For the simulation results of weak coupling, described in this section, the number of positive Lyapunov exponents is equal to the number of cells, which means hyperchaos is obtained for $L \geq 2$. This confirms the conjecture on the number of positive Lyapunov exponents made in [Kapitaniak \& Chua, 1994]. The estimated Lyapunov exponents are shown in Table 1. The algorithm with GramSchmidt orthonormalization described in [Parker \& Chua, 1989] has been implemented in Matlab with joint simulation of the system equation and its variational equation using a Runge-Kutta integration rule (ode23 in Matlab). The variational equations were analytically derived from Eq. (1). A Matlab to $\mathrm{C}$ compiler ( $m c c$ in Matlab) has been used. All simulations were done on a SUN Ultra1-140 workstation. The estimated values of the negative Lyapunov exponents much depend on the choice of the time horizon $T$ on which the system and its variational equation are simulated. Choosing a larger $T$ (but still making sure that no overflow occurs in the variational equation) results in a larger estimate for the Lyapunov dimension of the system. Indeed, according to Kaplan and Yorke the Lyapunov dimension is defined as $\mathcal{D}_{l}=j+\left(\lambda_{1}+\cdots+\lambda_{j}\right) /\left|\lambda_{j+1}\right|$ where $j$ is the largest integer such that $\lambda_{1}+\cdots+\lambda_{j} \geq 0$, provided the Lyapunov exponents are ordered as $\lambda_{1} \geq \cdots \geq \lambda_{d}$, with $d$ being the dimension of the state space [Parker \& Chua, 1989].

\section{Route from Synchronization to the Hypercube}

For the case $n=2$ and 2 cells with unidirectional coupling one obtains synchronization for $K<-1.3$ and instability for $K>0.38$. The route from synchronization to the hypercube goes through intermittency with occasional bursts to higher dimensions, as shown on Fig. 8 for the case of diffusive coupling with 3 cells. This phenomenon has been reported in [Kapitaniak \& Chua, 1994] as well. For the case of diffusive coupling and 3 cells, the array synchronizes approximately for $D_{y}>0.1$. For $D_{y}=0.1$ and $D_{y}=0.09$ the system behaves 'close' to synchronization, with the formation of additional scrolls in the common cell-space, but the array is not able to develop the full hypercube. For the simulations, the same initial states were taken as in the previous section. 


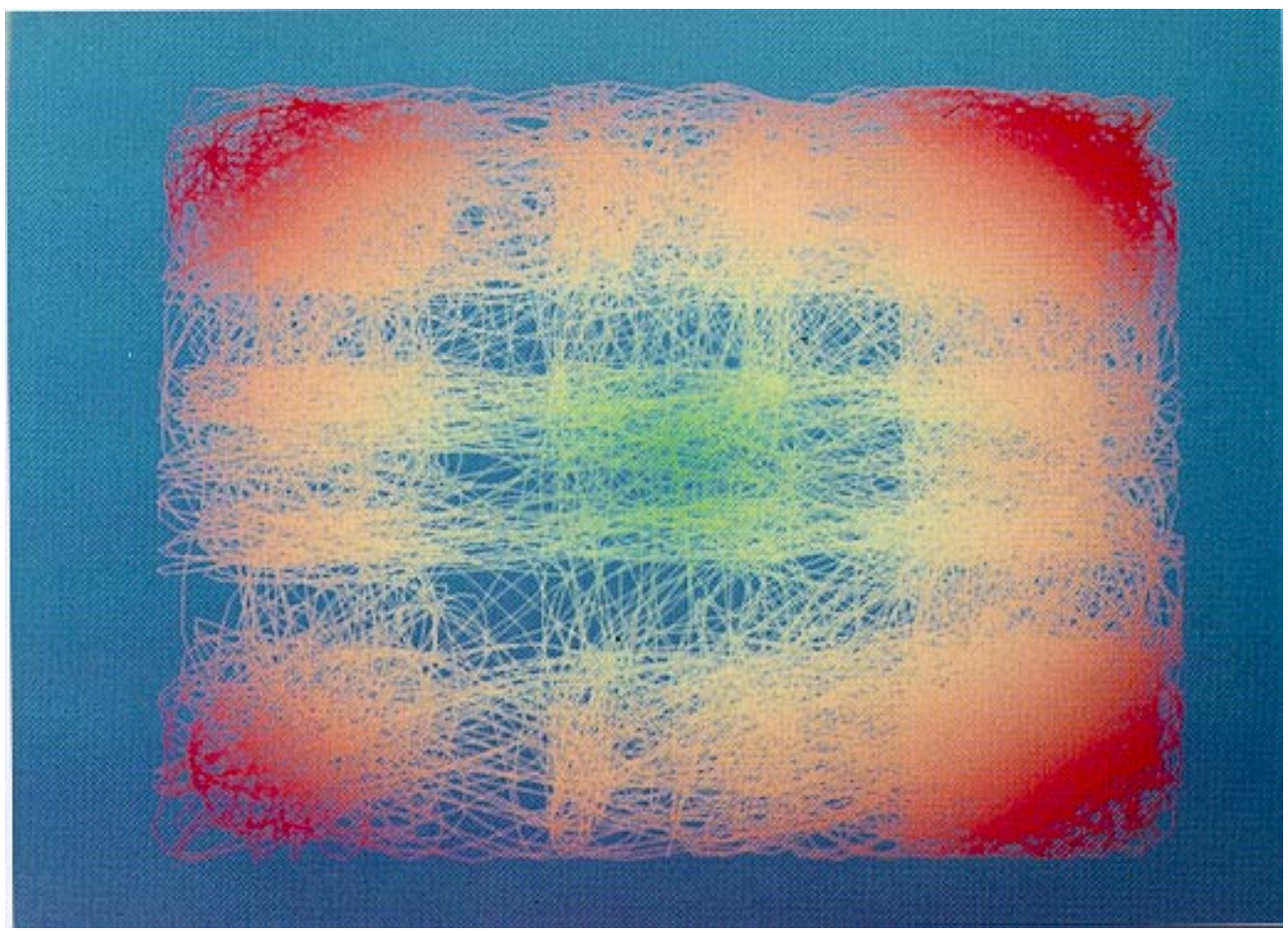

(a)

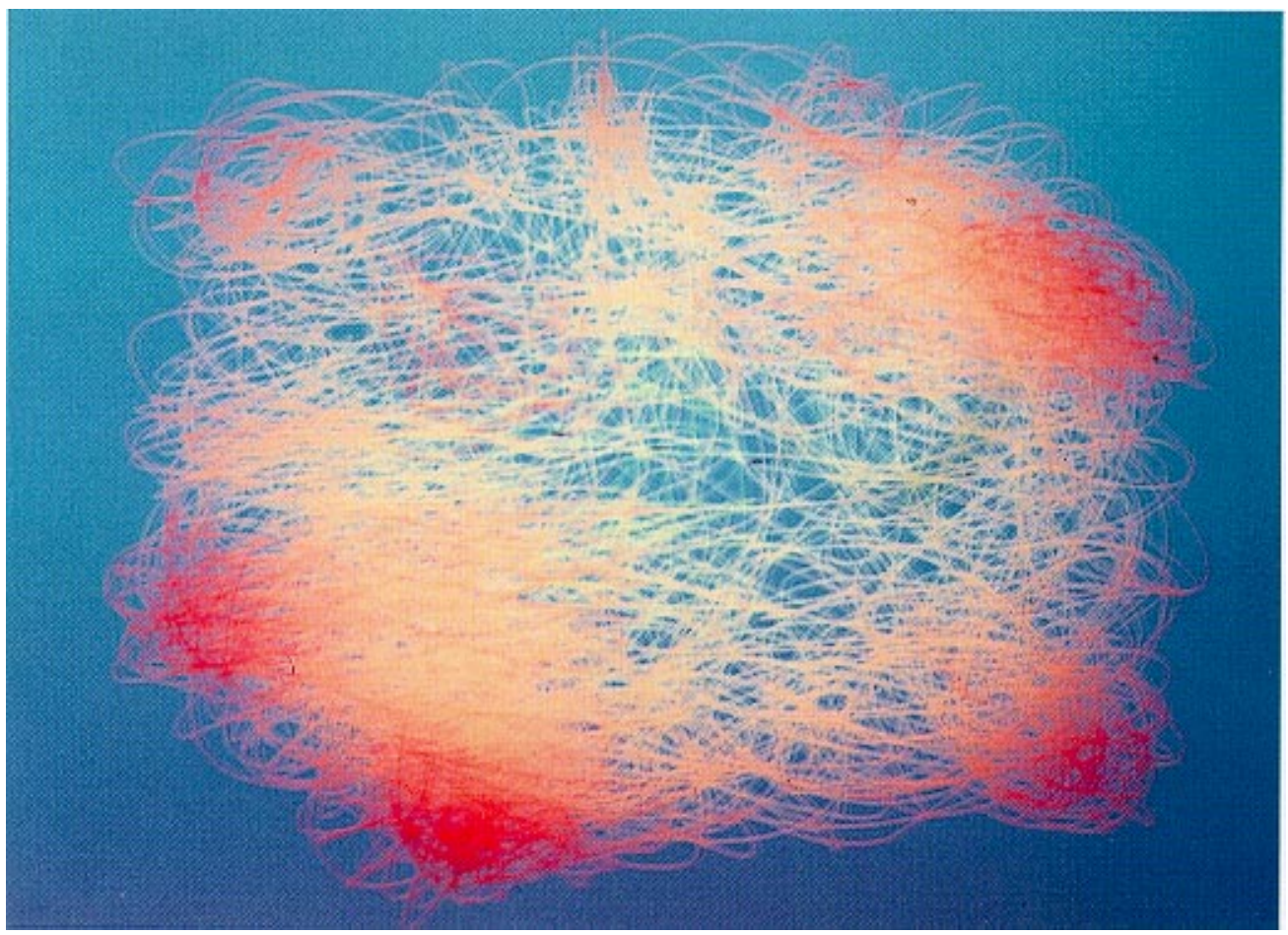

(b)

Fig. 2. (a) 2-double scroll square from a 1D-CNN with 2 cells of 2-double scroll circuits and unidirectional coupling between the cells $(K=0.001)$. Note the resemblance and difference with Fig. 2 in [Kapitaniak \& Chua, 1994]. Shown is the plane $\left(x^{(1)}, x^{(2)}\right)$. (b) 2-double scroll cube from a 1D-CNN with 3 cells of 2-double scroll circuits and unidirectional coupling between the cells $(K=0.001)$. The subspace $\left(x^{(1)}, x^{(2)}, x^{(3)}\right)$ is shown. 


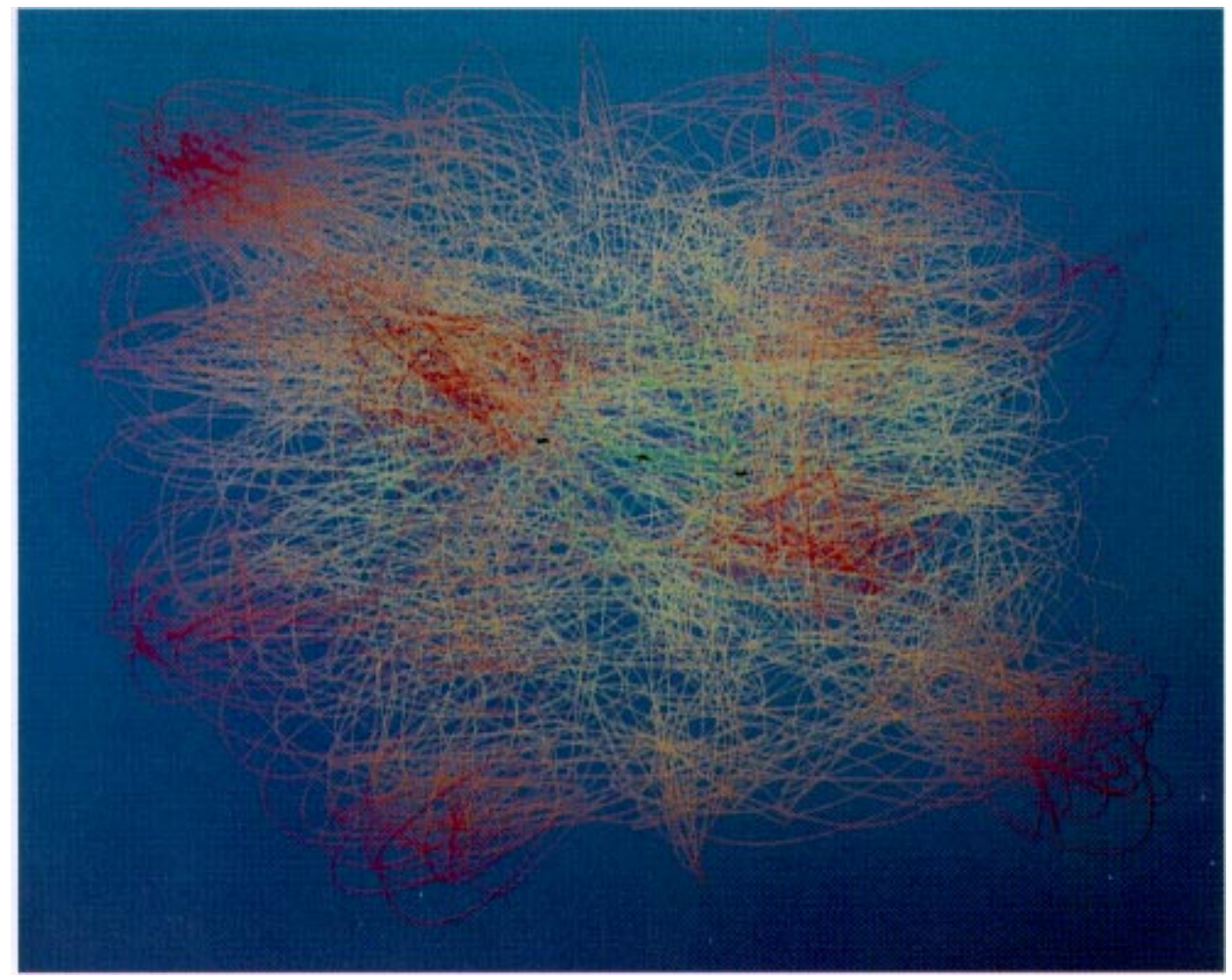

(a)

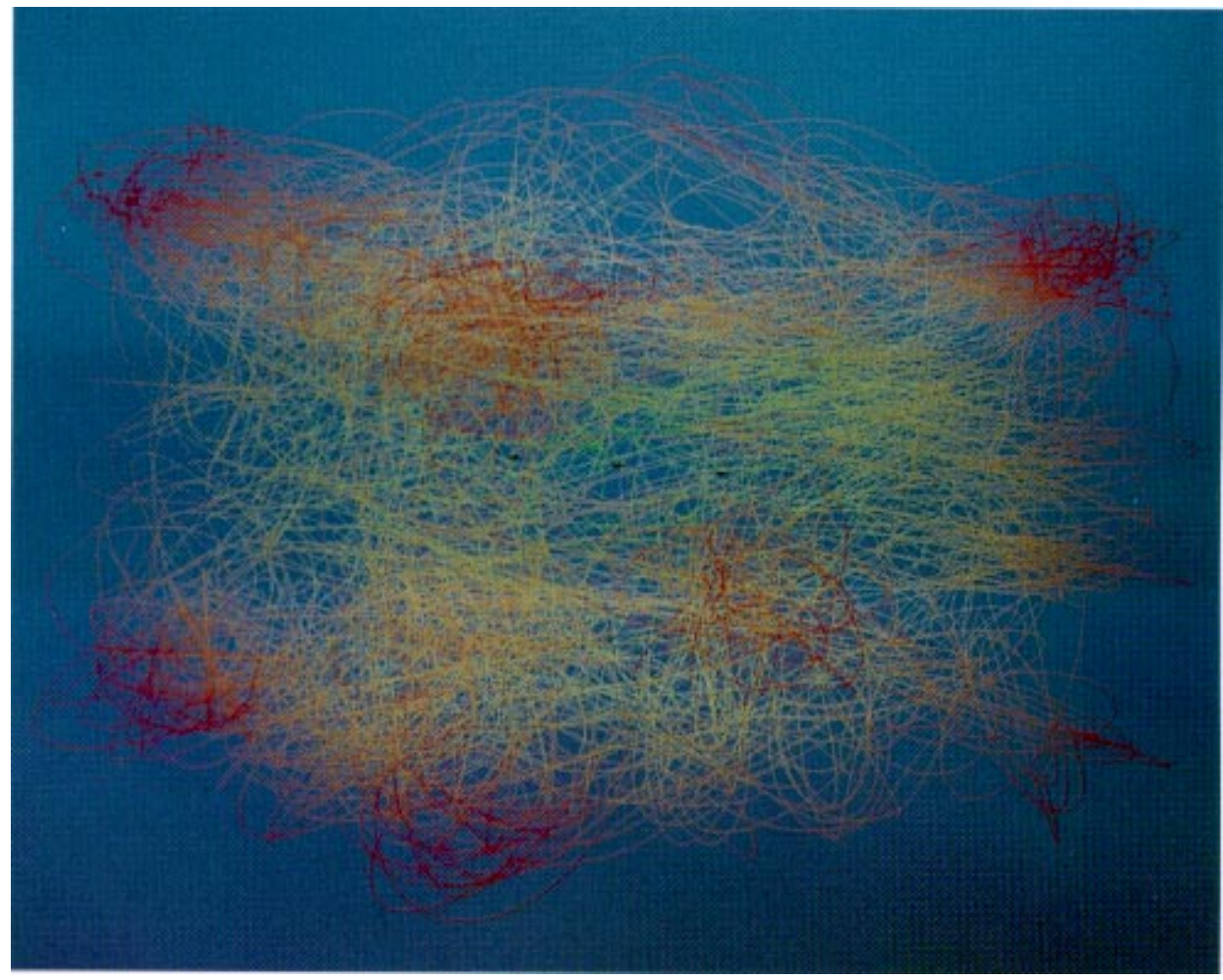

(b)

Fig. 3. 2-double scroll hypercube from a 1D-CNN with 4 cells of 2-double scroll circuits and diffusive coupling between the cells $\left(D_{y}=0.01\right)$. Two views are shown on this four-dimensional hypercube: $(\mathrm{a})\left(x^{(1)}, x^{(2)}, x^{(3)}\right) ;(\mathrm{b})\left(x^{(2)}, x^{(3)}, x^{(4)}\right)$. 


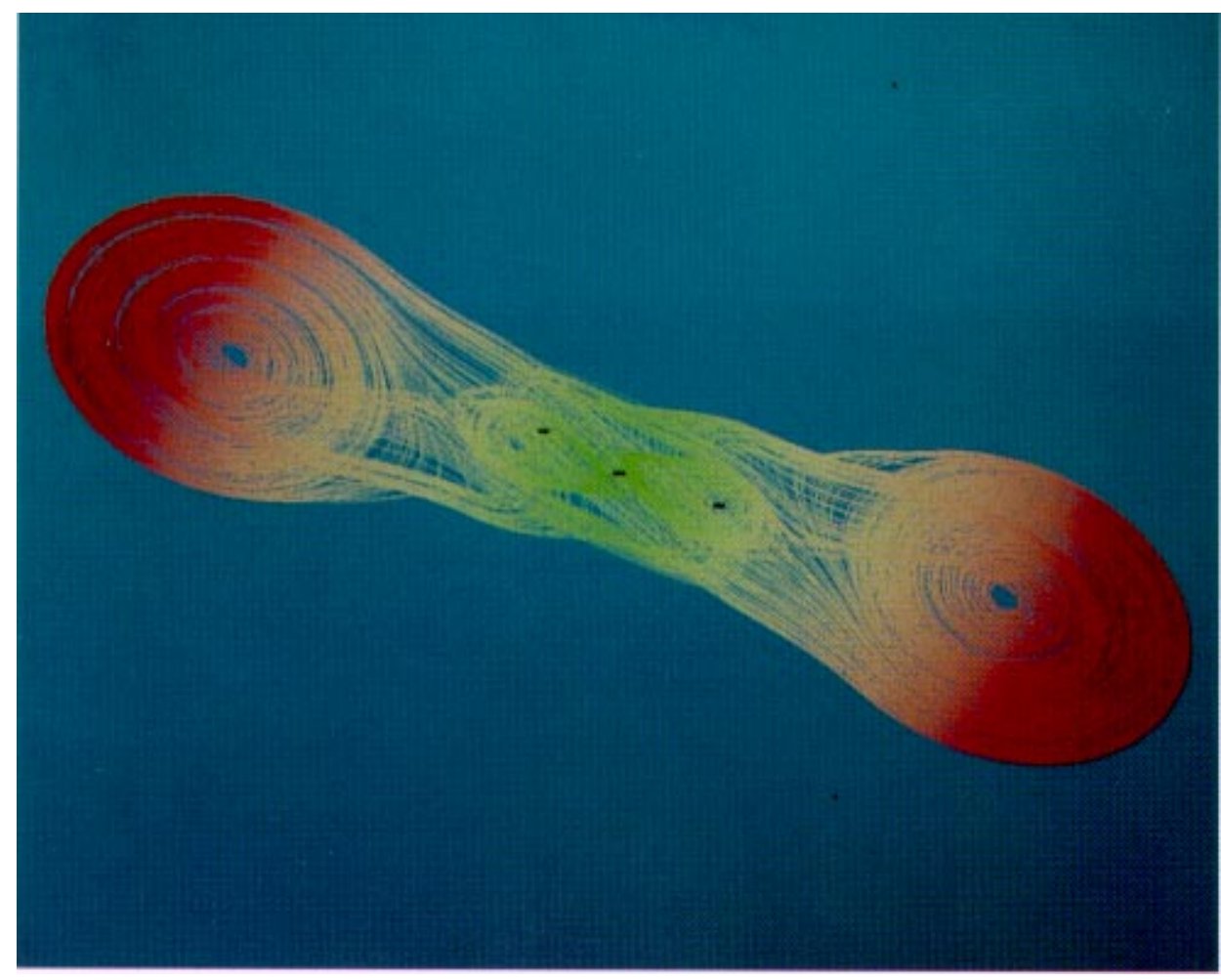

(a)

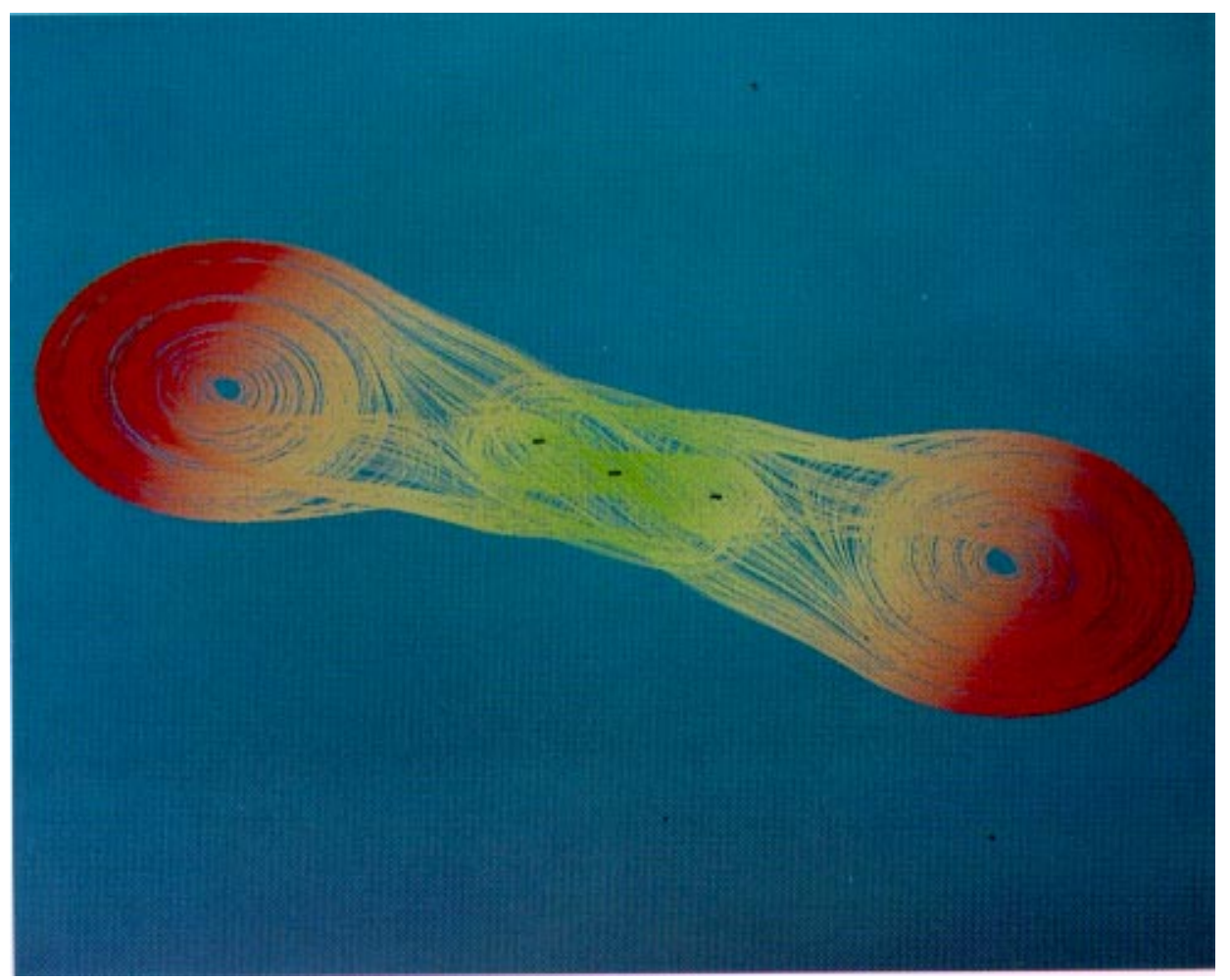

(b)

Fig. 4. While the 2-double scroll circuits reveal in their common cell state subspace a four-dimensional 2-double scroll hypercube (Fig. 3), the individual cells remain behaving as 2-double scrolls. In this figure this is illustrated for two of the four cells (cell 1 and 3): (a) $\left(x^{(1)}, y^{(1)}, z^{(1)}\right)$, (b) $\left(x^{(3)}, y^{(3)}, z^{(3)}\right)$. 

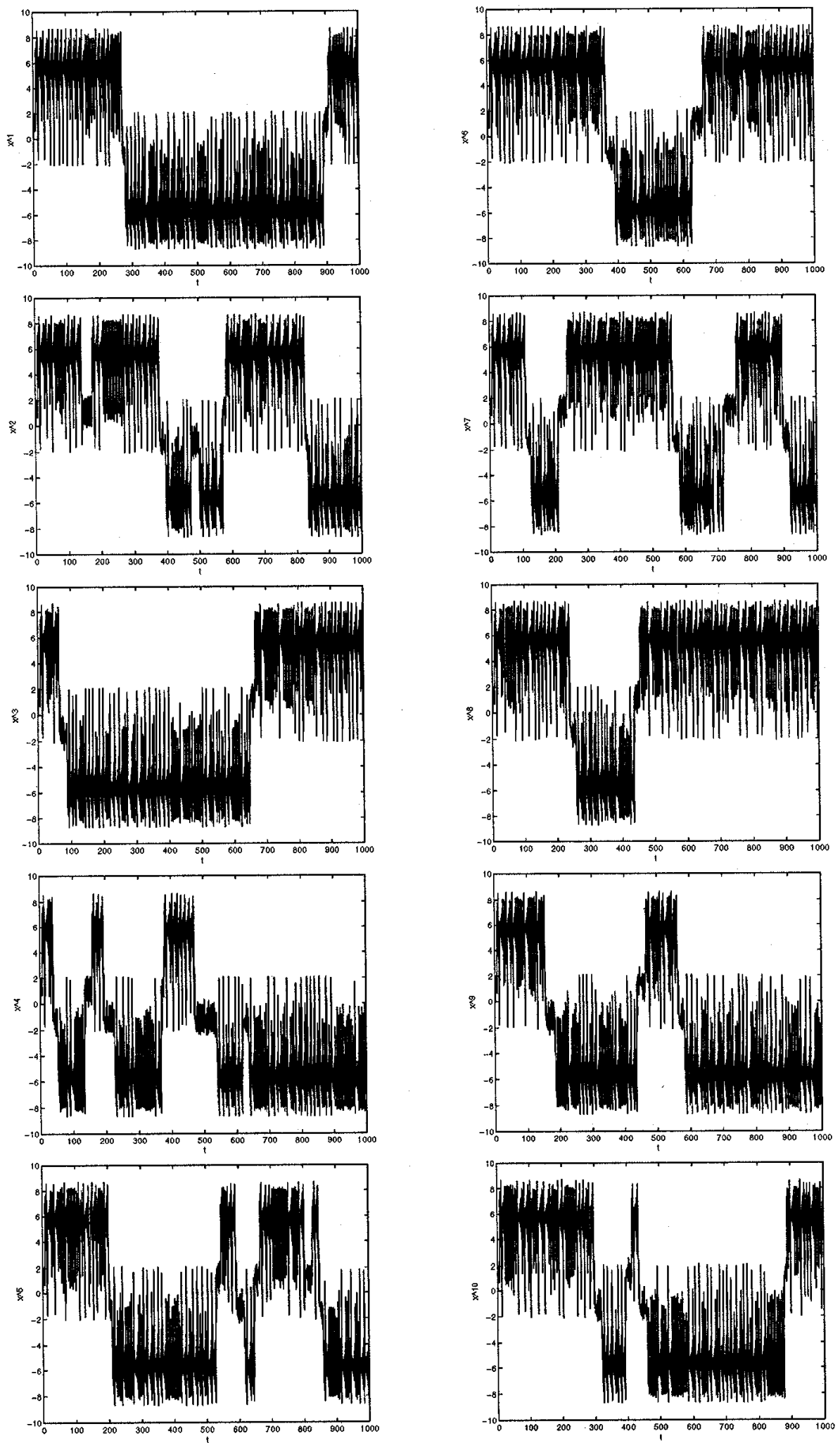

Fig. 5. Diffusive coupling $\left(D_{y}=0.001\right)$ between 10 cells of 2 -double scroll circuits in a 1 D-CNN, resulting into a tendimensional 2-double scroll hypercube. Shown are the variables $x^{(j)}$ for $j=1,2, \ldots, 10$ with respect to time for a certain initial state. 


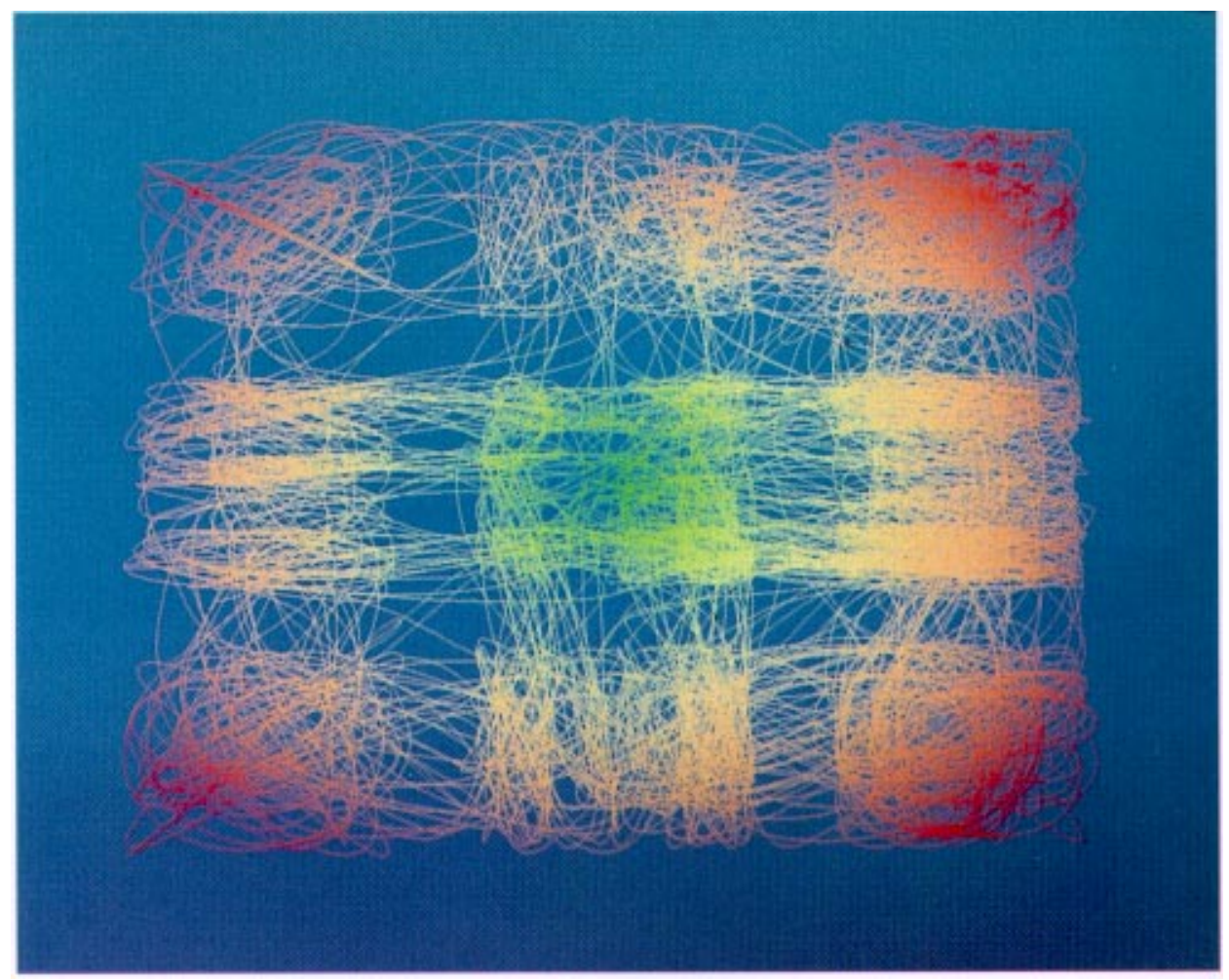

(a)

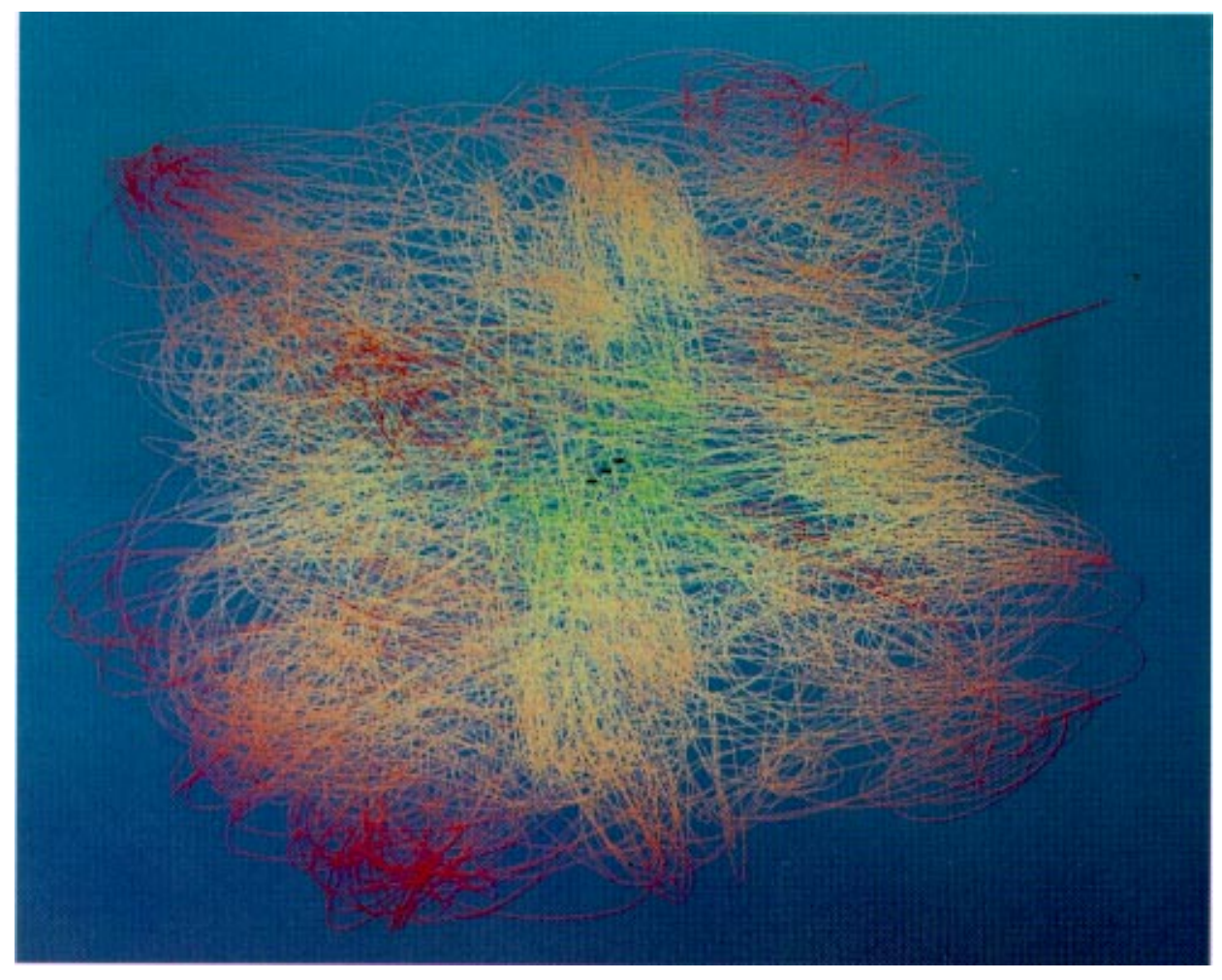

(b)

Fig. 6. (a) 3-double scroll square from a 1D-CNN with 2 cells of 3-double scroll circuits and unidirectional coupling between the cells $(K=0.01)$. Shown is the plane $\left(x^{(1)}, x^{(2)}\right)$. (b) 3 -double scroll cube from a 1D-CNN with 3 cells of 3-double scroll circuits and diffusive coupling between the cells $\left(D_{x}=0.001\right)$. Shown is the view $\left(x^{(1)}, x^{(2)}, x^{(3)}\right)$. 


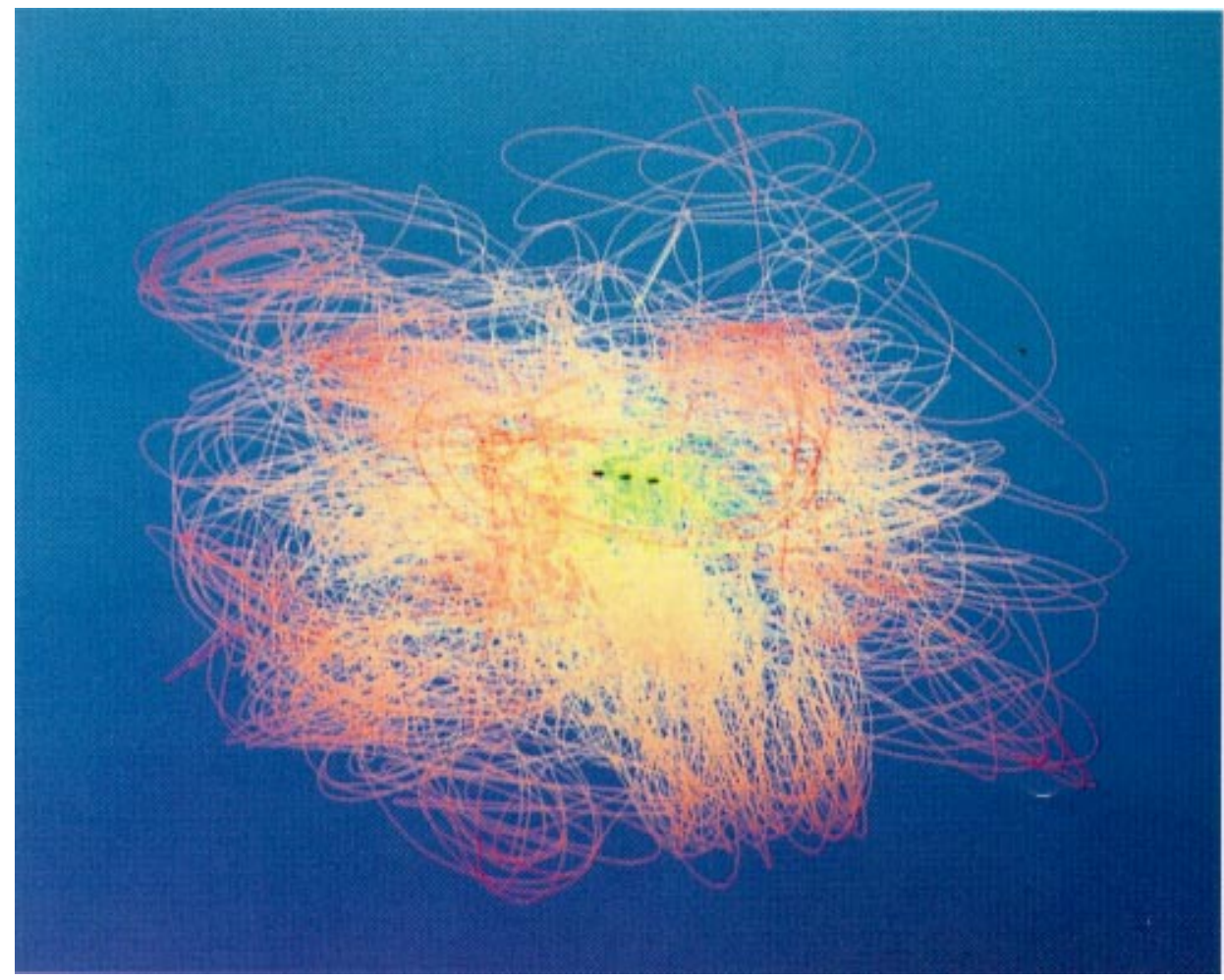

(a)

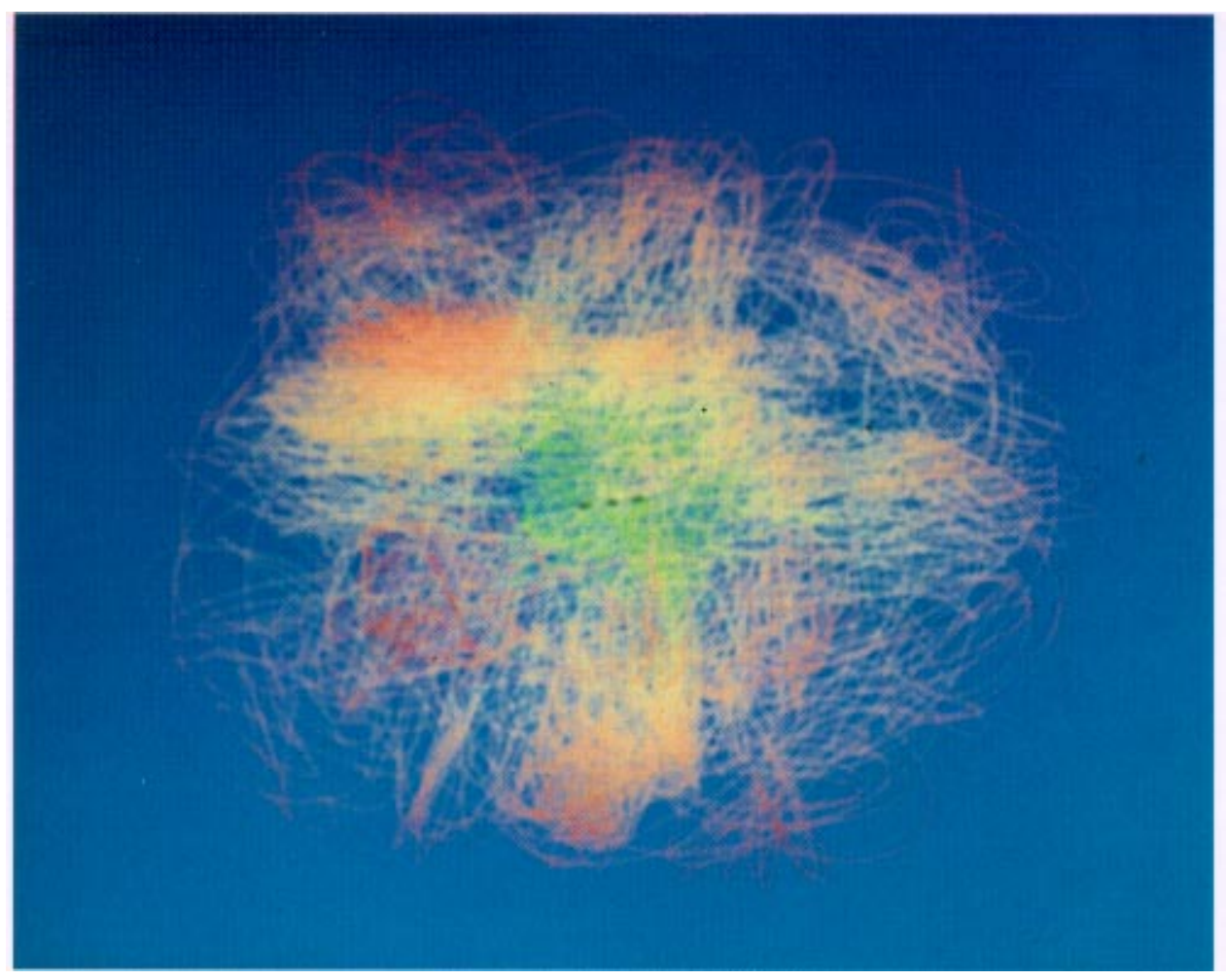

(b)

Fig. 7. 3-double scroll hypercube from a 1D-CNN with 4 cells of 3-double scroll circuits and diffusive coupling between the cells $(D=0.001)$. Two views are shown on this four-dimensional hypercube: (a) $\left(x^{(1)}, x^{(2)}, x^{(3)}\right) ;(\mathrm{b})\left(x^{(2)}, x^{(3)}, x^{(4)}\right)$. 
Table 1. In this Table estimations of Lyapunov exponents are made for $1 \mathrm{D}-\mathrm{CNNs}$ with 2- and 3-double scroll cells and different numbers of cells. For weak coupling the array has a number of positive $\left(\lambda_{+}\right)$, zero $\left(\lambda_{0}\right)$ and negative $\left(\lambda_{-}\right)$Lyapunov exponents equal to the number of cells $L . \mathcal{D}_{l}$ is the estimated Lyapunov dimension and $T$ is the time horizon on which the array together with its variational equation have been simulated.

\begin{tabular}{|c|c|c|c|c|c|c|}
\hline Array & Coupling & $\lambda_{+}$ & $\lambda_{0}$ & $\lambda_{-}$ & $\mathcal{D}_{l}$ & $T$ \\
\hline \multirow[t]{2}{*}{$n=2, L=2$} & $K=0.001$ & 0.30 & 0.00 & -0.87 & 4.6 & 30 \\
\hline & & 0.28 & 0.00 & -0.90 & & \\
\hline \multirow[t]{4}{*}{$n=2, L=4$} & $D_{y}=0.01$ & 0.34 & 0.00 & -1.41 & 8.7 & 20 \\
\hline & & 0.28 & 0.00 & -1.43 & & \\
\hline & & 0.23 & 0.00 & -1.46 & & \\
\hline & & 0.17 & 0.00 & -1.48 & & \\
\hline \multirow[t]{10}{*}{$n=2, L=10$} & $D_{y}=0.001$ & 0.34 & 0.00 & -2.62 & 21.1 & 10 \\
\hline & & 0.32 & 0.00 & -2.77 & & \\
\hline & & 0.31 & 0.00 & -2.86 & & \\
\hline & & 0.31 & 0.00 & -2.92 & & \\
\hline & & 0.29 & 0.00 & -2.96 & & \\
\hline & & 0.28 & 0.00 & -2.99 & & \\
\hline & & 0.27 & 0.00 & -3.02 & & \\
\hline & & 0.26 & 0.00 & -3.04 & & \\
\hline & & 0.26 & 0.00 & -3.07 & & \\
\hline & & 0.23 & 0.00 & -3.10 & & \\
\hline \multirow[t]{2}{*}{$n=3, L=2$} & $K=0.01$ & 0.28 & 0.00 & -0.88 & 4.6 & 30 \\
\hline & & 0.27 & 0.00 & -0.91 & & \\
\hline \multirow[t]{4}{*}{$n=3, L=4$} & $D_{x}=0.001$ & 0.33 & 0.00 & -1.42 & 8.8 & 20 \\
\hline & & 0.29 & 0.00 & -1.43 & & \\
\hline & & 0.25 & 0.00 & -1.47 & & \\
\hline & & 0.23 & 0.00 & -1.49 & & \\
\hline
\end{tabular}

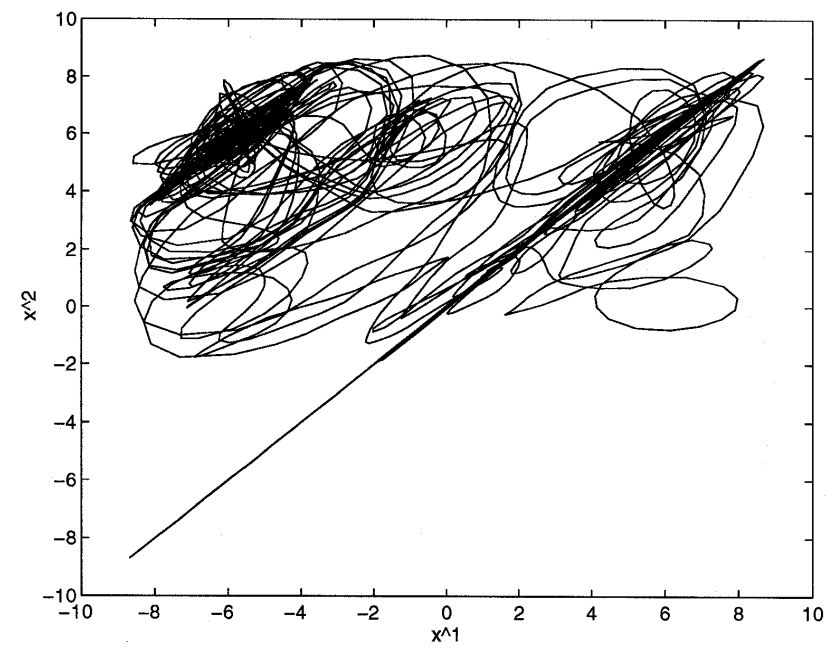

(a)

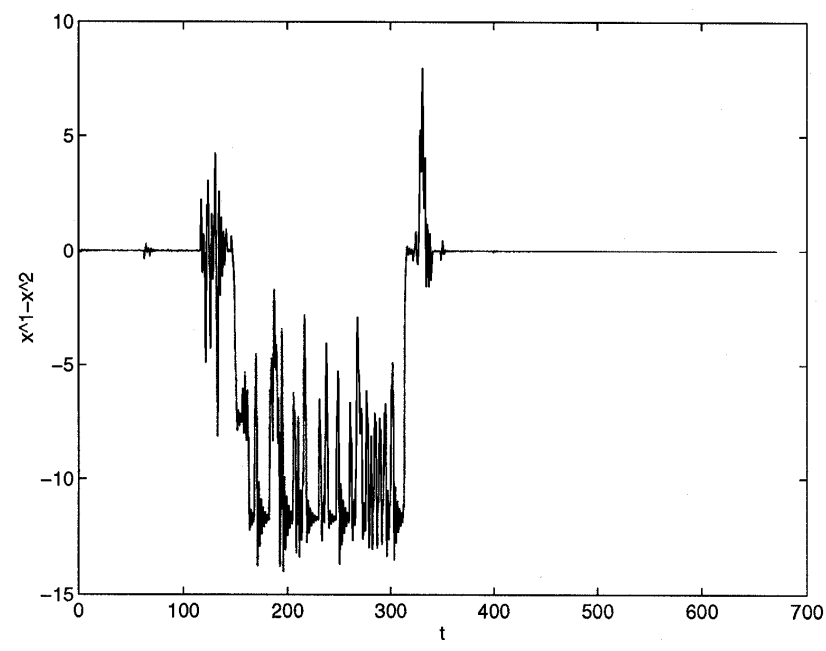

(b)

Fig. 8. This figure shows unidirectional coupling between two 2-double scroll circuits for $K=-1.2$. Occasional synchronization between the two cells is clearly visible on (a) $\left(x^{(1)}, x^{(2)}\right)$ and $(\mathrm{b}) x^{(1)}(t)-x^{(2)}(t)$. 


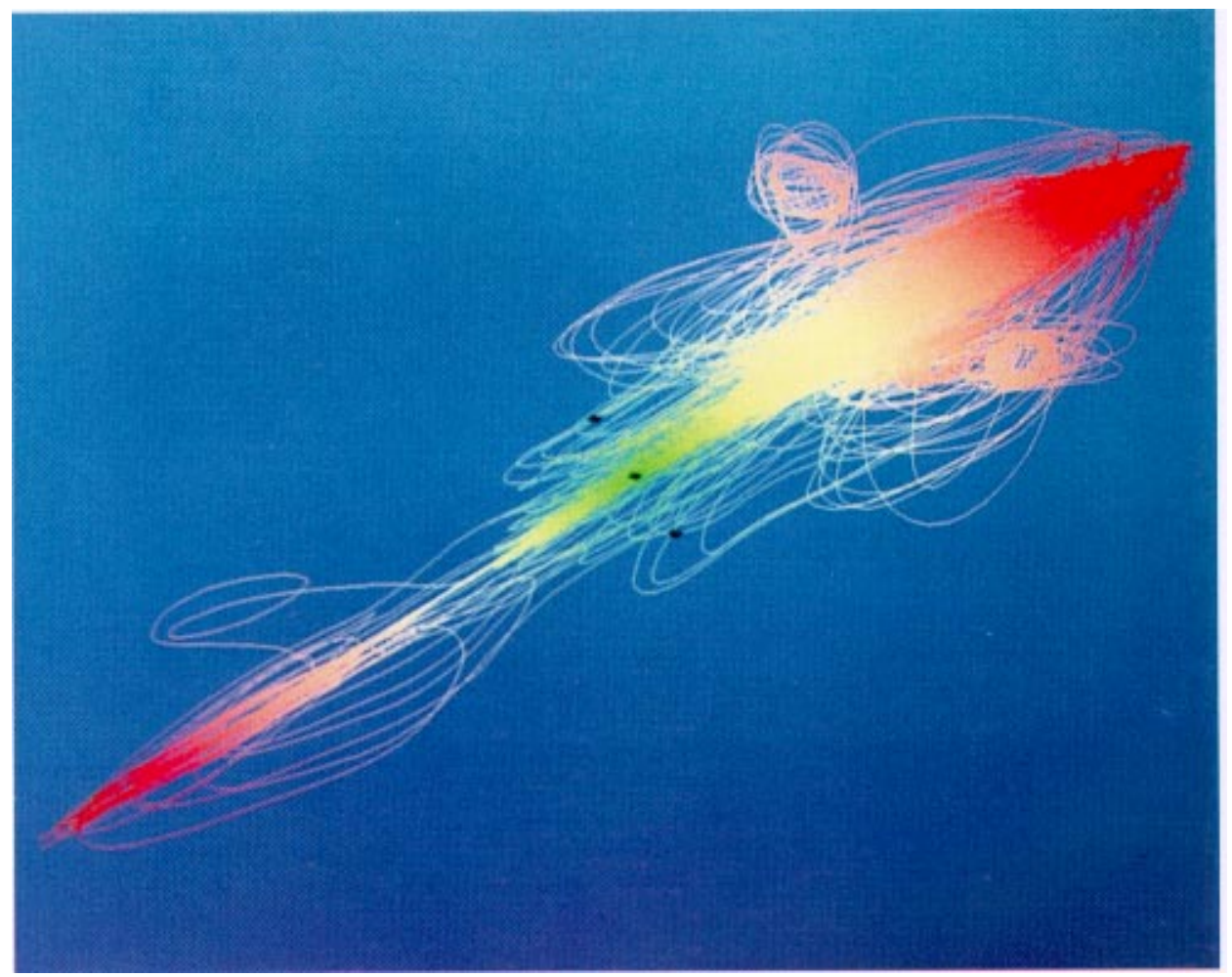

(a)

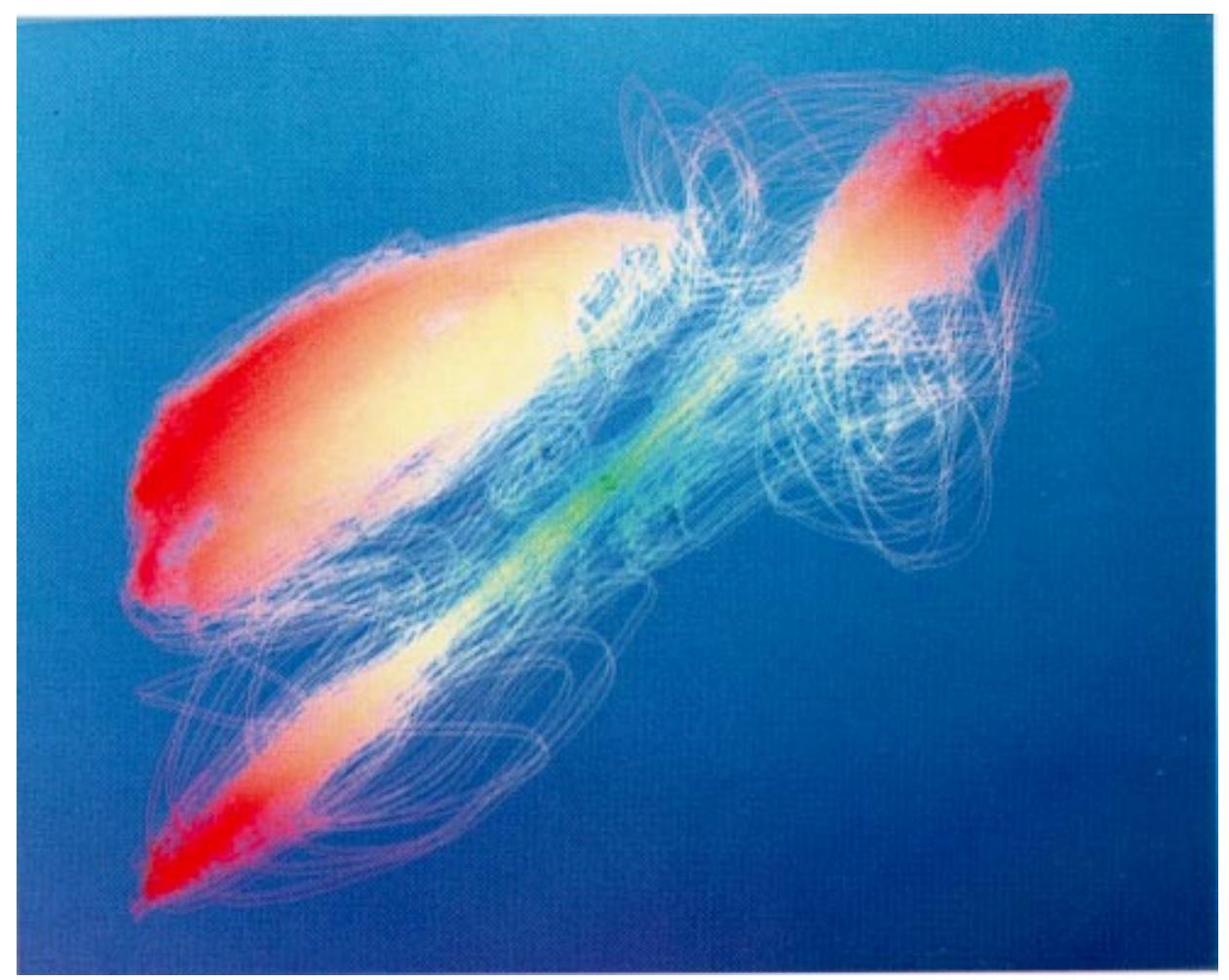

(b)

Fig. 9. Diffusive coupling between three 2-double scroll circuits for (a) $D_{y}=0.1$ and (b) $D_{y}=0.09$. For this choice of parameters the system is not able to develop the 2-double scroll cube. Shown is the view $\left(x^{(1)}, x^{(2)}, x^{(3)}\right)$. 


\section{Conclusion}

In this paper the new phenomenon of $n$-double scroll hypercubes has been described, which occurs in weak unidirectional or diffusive coupling of $n$ double scroll cells in one-dimensional CNNs. The dimension of the hypercube is equal to the number of cells in the array. The hypercube is filled with a number of multiple scrolls that increases with the number of $n$. For weak coupling the individual cells remain behaving as $n$-double scrolls, while the hypercube is formed in the common cell-space. For the simulations that have been done, the array behaves hyper-chaotically with a number of positive Lyapunov exponents equal to the number of cells. The route from synchronization to the hypercube in the array goes by means of intermittency, as earlier described by Kapitaniak and Chua for unidirectional coupling of Chua's circuits in 1-D CNNs. Potential applications of the $n$-double scroll hypercube CNN are in the use of high-dimensional chaos for secure communication applications and artificial neural information processing systems.

\section{Acknowledgments}

This research work was carried out at the University of California at Berkeley, in the framework of the Belgian Programme on Interuniversity Poles of Attraction, initiated by the Belgian State, Prime Minister's Office for Science, Technology and Culture (IUAP-17) and in the framework of a Concerted Action Project MIPS (Modelbased Information Processing Systems) of the Flemish Community. The work is supported in part by the Office of Naval Research under grant N00014-96-1-0753. The authors would like to thank Tao Yang and Chai Wah Wu for helping to produce the color pictures.

\section{References}

Arena, P., Baglio, P., Fortuna, F. \& Manganaro, G. [1996] "Generation of $n$-double scrolls via cellular neural networks," Int. J. Circuits Theory Appl. 24, $241-252$.

Chua, L. O., Komuro, M. \& Matsumoto, T. [1986] "The double scroll family," IEEE Trans. Circuits Syst. - I 33(11), 1072-1118.
Chua, L. O. \& Roska, T. [1993] "The CNN paradigm," IEEE Trans. Circuits Syst. - I 40(3), 147-156.

Chua, L. O. [1994] "Chua's circuit 10 years later," Int. J. Circuits Theory Appl. 22, 279-305.

Chua, L. O., Hasler, M., Moschytz, G. S. \& Neirynck, J. [1995] "Autonomous cellular neural networks: A unified paradigm for pattern formation and active wave propagation," IEEE Trans. Circuits Syst. - I 42(10), $559-577$.

Chua, L. O. \& Goraş, L. [1995] "Turing patterns in cellular neural networks," Int. J. Electron. 79(6), 719-736.

Kapitaniak, T., Chua, L. O. \& Zhong, G.-Q. [1994] "Experimental hyperchaos in coupled Chua's circuits," IEEE Trans. Circuits Syst. - I 41(7), 499-503.

Kapitaniak, T. \& Chua, L.O. [1994] "Hyperchaotic attractors of unidirectionally-coupled Chua's circuits," Int. J. Bifurcation and Chaos 4(2), 477-482.

Madan, R. N. (Guest ed.) [1993] Chua's Circuit: A Paradigm for Chaos (World Scientific Publishing, Singapore).

Nekorkin, V. I. \& Chua, L. O. [1993] "Spatial disorder and wave fronts in a chain of coupled Chua's circuits," Int. J. Bifurcation and Chaos 3(5), 1281-1291.

Parker, T. S. \& Chua, L. O. [1989] Practical Numerical Algorithms for Chaotic Systems (Springer-Verlag, New York).

Pérez-Muñuzuri, A., Pérez-Muñuzuri, V., Pérez-Villar, V. \& Chua, L. O. [1993] "Spiral waves on a 2-D array of nonlinear circuits," IEEE Trans. Circuits Syst. - I 40(11), 872-877.

Pivka, L. [1995] "Autowaves and spatio-temporal chaos in CNNs-Part I \& Part II: A tutorial," IEEE Trans. Circuits Syst. - I 42(10), 638-649 \& 650-664.

Pivka, L., Zheleznyak, A. L., Wu, C. W. \& Chua, L. O. [1995] "On the generation of scroll waves in a threedimensional discrete active medium," Int. J. Bifurcation and Chaos 5(1), 313-320.

Suykens, J. A. K. \& Vandewalle, J. [1993] "Generation of $n$-double scrolls $(n=1,2,3,4, \ldots)$," IEEE Trans. Circuits Syst. - I 40(11), 861-867.

Suykens, J. A. K. \& Vandewalle, J. [1997] "Master-slave synchronization of Lur'e systems," Int. J. Bifurcation and Chaos 7(3), 665-669.

Wu, C. W. \& Chua, L. O. [1994] "A unified framework for synchronization and control of dynamical systems," Int. J. Bifurcation and Chaos 4(4), 979-989.

Zheleznyak, A. L. \& Chua, L. O. [1994] "Coexistence of low-and high-dimensional spatiotemporal chaos in a chain of dissipatively coupled Chua's circuits," Int. J. Bifurcation and Chaos 4(3), 639-674. 